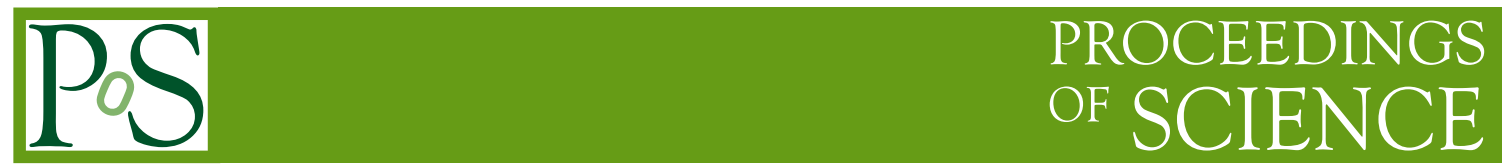

\title{
Extra dimensions and micro black holes at the LHC
}

\author{
Viatcheslav Valuev** \\ University of California, Los Angeles \\ 405 Hilgard Avenue, Los Angeles, CA 90095-1547, USA \\ E-mail: Viatcheslav.Valuev@cern.ch
}

We present recent studies of the ATLAS and CMS Collaborations on the potential to discover extra dimensions and microscopic black holes in the forthcoming 2009-10 LHC run. We show that there is a good chance that with an integrated luminosity of $\sim 100 \mathrm{pb}^{-1}$ accumulated at a center-of-mass energy of $10 \mathrm{TeV}$ the searches for such new physics will be extended beyond the existing limits.

European Physical Society Europhysics Conference on High Energy Physics, EPS-HEP 2009, July 16 - 222009

Krakow, Poland

\footnotetext{
* Speaker.

†n behalf of the ATLAS and CMS Collaborations.
} 


\section{Introduction}

Many theories beyond the Standard Model (SM) invoke extra dimensions (EDs) to explain why gravity is so much weaker than the other forces. The most popular scenarios of extra dimensions include ADD [1], in which the gravitational force is diluted by propagating through extra dimensions of macroscopic size, and RS [2], in which gravity originates on a hidden 4D brane and is suppressed away from the brane along the ED due to the warping of the brane. In these models, the strength of gravity is strongly increased at small distances, which opens up the possibility of observing quantum-gravity effects in the $\mathrm{TeV}$ energy range, in particular the production of microscopic black holes [3]. In this talk, we focus on the prospects to discover these phenomena in the ATLAS and CMS experiments [4] in the forthcoming 2009-10 LHC run, and on the main experimental challenges of such searches.

\section{Search for dilepton decays of new high-mass resonances}

Both the ADD and RS scenarios are expected to manifest themselves through modifications in the mass spectrum of high-mass dilepton pairs. While the closely-spaced Kaluza-Klein modes of the ADD model give rise to non-resonant distortions of the spectrum, the gravitons $\left(G^{*}\right)$ of the RS scenario would be seen as narrow resonances. The dominant and irreducible background is the Drell-Yan production of lepton pairs. Other backgrounds can be subdivided into two categories: I) with two high- $p_{\mathrm{T}}$ isolated leptons $(t \bar{t}, t W, W W)$; II) with one or more leptons produced in a jet $(W+$ jets, dijets, $\gamma+$ jets). Type-I backgrounds are expected to be measured from data, using samples of $e \mu$ and $b$-tagged events. Type-II backgrounds can be efficiently suppressed by isolation and kinematic cuts and their residual contribution evaluated from data, using the measured probability for a jet to fake a lepton as well as same-sign $\left(e^{+} e^{+}, \mu^{-} \mu^{-}\right.$, etc. $)$events.

Figure 1a shows the simulated dielectron invariant-mass spectrum for the SM backgrounds and a $1 \mathrm{TeV}$ resonance corresponding to an integrated luminosity of $100 \mathrm{pb}^{-1}$ accumulated at a centerof-mass energy $(\sqrt{s})$ of $10 \mathrm{TeV}$. In order to minimize sensitivity to uncertainties in the expected signal and background levels, the fit to the data explores the difference in shape between the signal "bump" and the steeply-falling background. Evaluations of the statistical significance show that an integrated luminosity of $100 \mathrm{pb}^{-1}$ at $\sqrt{s}=10 \mathrm{TeV}$ should be sufficient to probe the RS $G^{*}$ mass region of up to 1.3-1.5 TeV in each leptonic decay channel [5, 6].

\section{Search for extra dimensions in the diphoton channel}

Since the graviton is a spin-2 particle, its decays to diphotons can proceed via an s-wave process, resulting in a larger branching ratio compared to that of difermion decays. The dominant and irreducible background is the SM production of diphotons, which mainly occur through $t$ channel processes. The shape of this background at high invariant masses is obtained from a detailed Monte Carlo (MC) simulation, whereas its absolute normalization comes from normalizing the MC-based spectrum to data at low diphoton masses, where the contribution from possible signal is negligible. Other backgrounds include jets faking photons in $\gamma+$ jet and dijet events and electrons from Drell-Yan events misidentified as photons; their contributions can be measured in samples of identified jets and $Z \rightarrow e e$ events, respectively. 

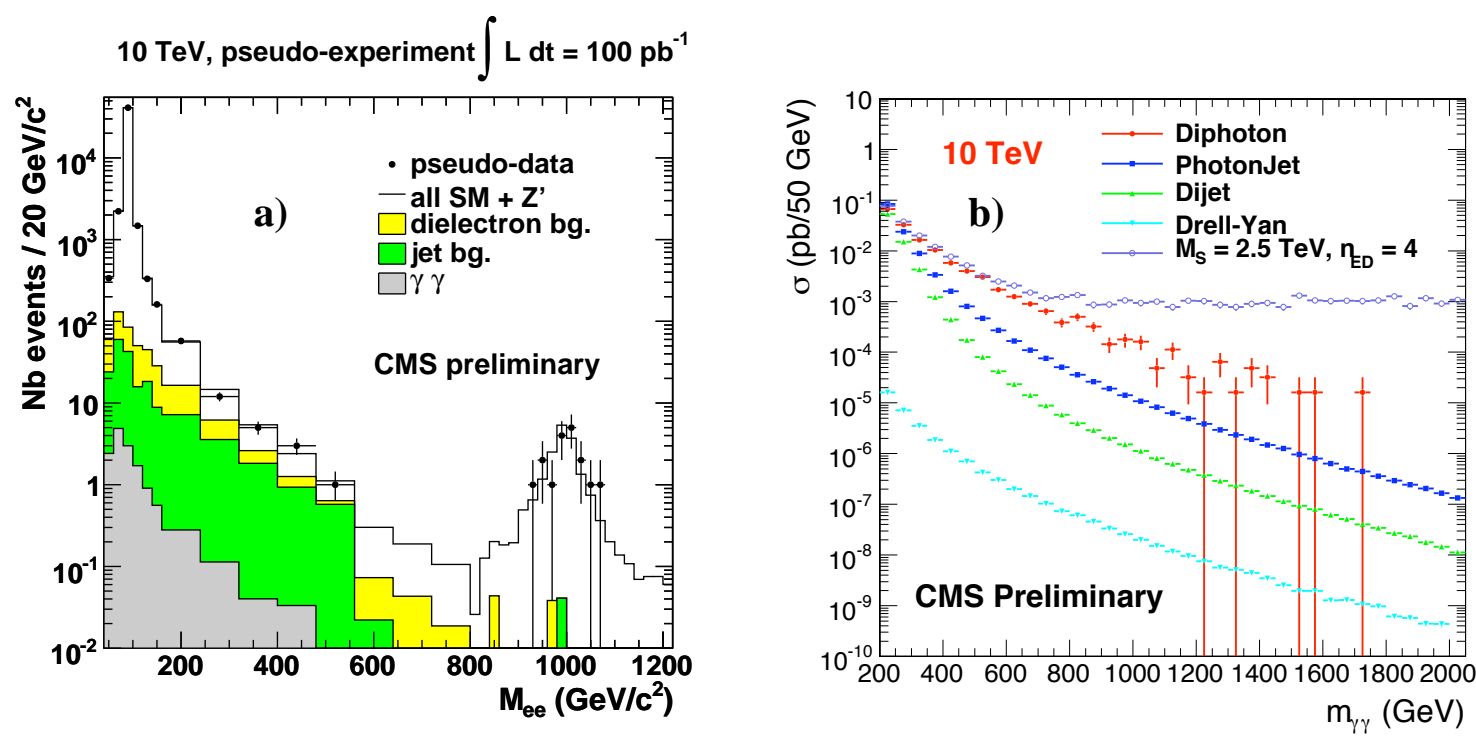

Figure 1: a) A simulated dielectron invariant-mass spectrum for an integrated luminosity of $100 \mathrm{pb}^{-1}$ at $\sqrt{s}$ $=10 \mathrm{TeV}$. The shaded areas show contributions from various non-Drell-Yan backgrounds; the non-shaded histogram gives the sum of all SM backgrounds and the signal from an additional heavy neutral gauge boson of the Sequential Standard Model $\left(Z_{\mathrm{SSM}}\right)$ with a mass of $1 \mathrm{TeV}$; points with error bars show the outcome of a single signal-plus-background MC experiment. b) The expected composition of the diphoton invariant-mass spectrum at $\sqrt{s}=10 \mathrm{TeV}$. The distribution at the top shows the sum of the ADD signal (in a model with ultraviolet cutoff value $M_{S}$ of $2.5 \mathrm{TeV}$ and with four EDs) and the SM direct diphoton background.

The optimal sensitivity to the ED signal is obtained by selecting events with two high- $E_{\mathrm{T}}$ photons, each in the pseudorapidity region of $\left|\eta_{\gamma}\right|<1$.5. Figure $1 \mathrm{~b}$ shows the expected contributions from various SM backgrounds as well as the ADD signal to the diphoton invariant-mass spectrum at $\sqrt{s}=10 \mathrm{TeV}$, for events passing all selection criteria. One searches for an excess of signal over the background in the mass range above $700 \mathrm{GeV}$. Evaluations of the ADD discovery potential show that lower limits on the effective Planck scale of up to a factor of two higher than the current best limits can be obtained with an integrated luminosity of $100 \mathrm{pb}^{-1}$ [7]. The sensitivity to the parameters of the RS model is expected to be similar to that in the dilepton channels [8].

\section{Search for large extra dimensions in mono-jets}

Another way to probe models of large EDs such as ADD, complementary to the searches for virtual graviton decays described above, is to look for real gravitons recoiling against a quark or a gluon. The signal signature consists of large missing $E_{\mathrm{T}}$ back-to-back to a high- $p_{\mathrm{T}}$ jet. The dominant SM backgrounds are $Z+$ jets with the $Z$ decaying into neutrinos, $W+$ jets followed by a leptonic $W$ decay, and QCD events. The main signal-background discriminator is the $M H T$ variable, defined as the negative vector sum of $p_{\mathrm{T}}$ of all high- $p_{\mathrm{T}}$ jets. The optimal sensitivity to the ED signal is obtained by selecting events with no isolated leptons, one or two high- $p_{\mathrm{T}}$ jets with $\left|\eta_{\text {jet }}\right|<1.7$, and $|M H T|>250 \mathrm{GeV}$. The projected discovery reach in this channel is about the same as in the diphoton channel [9]. 


\section{Search for micro black holes}

If microscopic black holes are produced at the LHC, they should undergo Hawking evaporation: particle pairs produced by vacuum fluctuations may be split, with one particle falling into the black hole and the other projected outward. This gives a very distinct signature characterized by a large number of high- $p_{\mathrm{T}}$ final-state particles and jets and large missing $E_{\mathrm{T}}$. The main sources of the background are $t \bar{t}$, dijet and $W+$ jet events. A pure sample of signal events can be obtained by selecting events containing at least one high- $p_{\mathrm{T}}$ lepton and with large $\sum\left|p_{\mathrm{T}}\right|$ of all reconstructed objects. The generation of micro black holes involves many assumptions, but if the predicted signature is correct, it should be detectable at the LHC [10].

\section{Conclusion}

We presented some recent studies by the ATLAS and CMS Collaborations dedicated to searches for extra dimensions and micro black holes in the forthcoming 2009-10 LHC run. The focus of the analyses is currently on data-driven methods of estimating backgrounds and efficiencies, and on techniques to minimize systematic uncertainties. The scenario in which an integrated luminosity of $\sim 100 \mathrm{pb}^{-1}$ is accumulated at $\sqrt{s}=10 \mathrm{TeV}$ offers good chances to go beyond the existing limits.

\section{References}

[1] N. Arkani-Hamed, S. Dimopoulos and G. Dvali, The hierarchy problem and new dimensions at a millimeter, Phys. Lett. B 429 (1998) 263.

[2] L. Randall and R. Sundrum, Large mass hierarchy from a small extra dimension, Phys. Rev. Lett. 83 (1999) 3370 .

[3] S.B. Giddings and S.D. Thomas, High energy colliders as black hole factories: The end of short distance physics, Phys. Rev. D 65 (2002) 056010 [hep-ph/ 0106219 ].

S. Dimopoulos and G.L. Landsberg, Black holes at the LHC, Phys. Rev. Lett. 87 (2001) 161602 [hep-ph/0106295].

[4] ATLAS Collaboration, The ATLAS experiment at the CERN LHC, JINST 3 (2008) S08003. CMS Collaboration, The CMS experiment at the CERN LHC, JINST 3 (2008) S08004.

[5] CMS Collaboration, Search for new high-mass resonances decaying to muon pairs in the CMS experiment, CMS PAS SBM-07-002 (2008). This and other CMS notes cited here are available at https://twiki.cern.ch/twiki/bin/view/CMS/PhysicsResults.

[6] CMS Collaboration, Search for high-mass resonances decaying into an electron pair in CMS at 10 TeV with $100 \mathrm{pb}^{-1}$, CMS PAS EXO-09-006 (2009).

[7] CMS Collaboration, Search for large extra dimensions in the diphoton final state, CMS PAS EXO-09-004 (2009).

[8] CMS Collaboration, Search for Randall-Sundrum gravitons in the diphoton final state, CMS PAS EXO-09-009 (2009).

[9] CMS Collaboration, Search for mono-jet final states from ADD extra dimensions at $\sqrt{\mathrm{s}}=10 \mathrm{TeV}$, CMS PAS EXO-09-013 (2009).

[10] ATLAS Collaboration, Expected performance of the ATLAS experiment: detector, trigger and physics, CERN-OPEN 2008-020 (2008) [arXiv: 0901 . 0512v4] . 